\title{
Plasma levels of VEGF-A, VEGF B, and VEGFR-1 and applicability of these parameters as tumor markers in diagnosis of breast cancer
}

\author{
Monika Zajkowska¹, Emilia Lubowicka², Paweł Malinowski ${ }^{3}$, Maciej Szmitkowski ${ }^{1}$ and \\ Sławomir Ławicki ${ }^{\bowtie}$
}

1Department of Biochemical Diagnostics, Medical University of Białystok, Białystok, Poland; ${ }^{2}$ Department of Esthetic Medicine, Medical University of Białystok, Białystok, Poland; '3Department of Oncological Surgery, Maria Sklodowska-Curie Oncology Center, Białystok, Poland

The VEGF family members are important factors in promoting angiogenesis and lymphangiogenesis in malignant processes. The aim of this study was to investigate plasma concentrations of VEGF-A, VEGF-B and their soluble VEGFR-1 receptor and their diagnostic utility and potency as compared to CA 15-3 in breast cancer patients and in relation to the control group. The study included 120 breast cancer patients and 60 control patients. Plasma levels of tested parameters were determined with ELISA and CA 15-3 levels were determined with CMIA. Concentrations of all tested parameters in breast cancer patients showed statistically significant difference when compared to the control groups (benign breast tumor patients and/ or healthy women). VEGF-B showed the highest values of sensitivity (Sn) and predictive value of a negative test result (NPV) in total BC group $(90 \%$ and $66.7 \%$, respectively) and, more importantly, in stages I-II of BC (SE: $86.8 \% ; 92.7 \%$, NPV: 82.8\%; 88.9\%, respectively). Among all parameters tested, VEGF-A showed the highest specificity (Sf) $(76.7 \%)$ and predictive value of a positive test result (PPV) $(84.8 \%)$, yet they were lower than for CA 15-3. VEGF-A was also the best parameter that had statistically significant Area Under Curve (AUC) in stages I (0.678) and II (0.768). In the whole group of BC patients all parameters tested showed statistically significant $A U C$, but the maximum range was obtained for the combination of VEGF-A and CA 15-3 (0.817). The combined analysis of the studied parameters and CA 15-3 resulted in an increase in sensitivity and AUC values, which provides hope for developing a new panel of biomarkers that may be used in BC diagnosis in the future.

Key words: vascular endothelial growth factors family members; diagnostic utility; area under curve; receiver operating characteristics

Received: 04 October, 2018; revised: 26 November, 2018; accepted: 04 December, 2018; vailable on-line: 13 December, 2018

e-mail: slawicki@umb.edu.pl

Abbreviations: AUC, area under curve; BC, breast cancer; CA 153 , cancer antigen 15-3; CMIA, chemiluminescent microparticle immunoassay; ELISA, enzyme-linked immunosorbent assay; NPV, negative predictive value; PPV, positive predictive value; ROC, receiver operating characteristics; $\mathrm{Sn}$, sensitivity; $\mathrm{Sf}$, specificity; VEGF-A, vascular endothelial growth factor $A$; VEGF-B, vascular endothelial growth factor B; VEGFR-1, vascular endothelial growth factor receptor 1

\section{INTRODUCTION}

Breast cancer (BC) is the most frequent cancer occurring in women worldwide (Torre et al., 2015) and the second leading cause of their death, also at an early age, in the United States (Oeffinger et al., 2015). Only in this country, 266,120 new BC cases were estimated in 2018 by American Cancer Society (Siegel et al., 2018). The most effective way to combat cancer is its prevention and early detection. Therefore, finding markers that would detect malignant cell transformation as early as possible is vital (Zajkowska et al., 2016).

Biochemical detection of this cancer type is currently limited to CA 15-3. Its prognostic relevance is supported by a number of studies, but it was shown that it has insufficient utility (diagnostic sensitivity and specificity) at less advanced stages of BC (Lawicki et al., 2004; Harris et al., 2007; Lawicki et al., 2016). Hence, a search for new markers that would exhibit higher diagnostic performance continues. Due to the fact that angiogenesis is a very important process involved in the development of tumor changes by enabling metastases and facilitating local development of cancer (Egeblad \& Werb, 2002), we predicted that the new candidates for tumor markers may be VEGF family members such as VEGF-A, VEGF-B, and their receptor - VEGFR-1.

VEGF-A was discovered in 1989. Its gene consists of 8 exons and plays an important role in the process of blood vessels forming (Leung et al., 1989; Żyła et al., 2014). It is synthesized by various cell types, including mast cells, smooth muscle cells in vessels, macrophages, fibroblasts, endothelial cells, monocytes, keratinocytes, eosinophils, T lymphocytes and cancer cells (Weryńska et al., 2009).

VEGF-B also stimulates angiogenesis in normal tissues, but its activity is much lower than VEGF-A. VEGF-B was first discovered in 1996. Its structure is very similar to the structure of VEGF-A, and in the mouse, VEGF-B shares approximately 43\% similarity with VEGF-A164 in the amino acid sequence (Weryńska et al., 2009). The Vegfb gene is highly conserved in mammals, exhibiting $88 \%$ homology between mouse and human growth factor at the amino acid sequence level (Bry et al., 2014).

There are three commonly known VEGF receptors (VEGFR-1, VEGFR-2, VEGFR-3). Each of them has the possibility of binding selected factors belonging to the VEGF family based on different affinities and selectivity (Carmeliet et al., 2013; Caballero et al., 2017). VEGFR-1 has been reported to bind VEGF-A 
and VEGF-B, mediate endothelial cell proliferation, monocyte and macrophage migration, and recruitment of endothelial cells from bone marrow and precursor hematopoietic cells (Takahashi, 2011).

The aim of the present study was to investigate plasma levels of the selected VEGF family members, their receptor, and a comparative tumor marker CA 15-3 in breast cancer patients, and to assess diagnostic utility (sensitivity, specificity, predictive values of positive and negative test results) and potency (ROC curve analysis) of these parameters in BC detection. In this study, healthy volunteers and women with benign breast lesions constituted one control group, which provided a more accurate reflection of the current female population. The data obtained in this study may prove the usefulness of the analyzed parameters (separately and together) as a new diagnostic panel in the detection of BC.

\section{MATERIAL AND METHODS}

Patients. Table 1 shows the tested groups. The study included 120 breast cancer patients (BC) diagnosed by the oncology group. The patients were treated in the Department of Oncology, Medical University, Białystok, Poland. Tumor classification and staging were conducted in accordance with the International Union Against Cancer Tumor-Node-Metastasis (UICC-TNM) classification.

In all cases, breast cancer histopathology was established by tissue biopsy of the mammary tumor or following surgery from tumor tissues (all patients with carcinoma ductale). The pretreatment staging procedures included physical and blood examinations, mammography, mammary ultrasound scanning, breast core biopsies and chest X-rays. In addition, radio isotopic bone scans, examination of bone marrow aspirates, and brain and chest CT scans were performed when necessary. None of the patients had received chemo- or radiotherapy prior to blood sample collection.

The control group included 60 patients: 28 with benign breast tumors (adenoma, fibroadenoma) and 32 healthy, untreated women who underwent mammary gland examination performed by a gynecologist prior to blood sample collection. In addition, mammary ultrasound scanning was performed in all cases. Benign breast tumor histopathology was established in all cases by tissue biopsy of the mammary tumor or after surgery.

For each of the patients qualified for the control group, the exclusion criteria such as: active infections and symptoms of an infection (both bacterial and viral), other comorbidities which can affect cytokine concentrations (respiratory diseases, digestive tract diseases) or systemic diseases such as lupus or rheumatoid arthritis, or collagenosis were applied.

Biochemical analyses. Venous blood samples were collected from each patient into an EDTA tube (S-Monovette, SARSTEDT, Germany), centrifuged $1000 \times \mathrm{g}$ for $15 \mathrm{~min}$ at $2-8^{\circ} \mathrm{C}$ to obtain plasma samples, and stored at $-85^{\circ} \mathrm{C}$ until assayed. The tested parameters were measured with the enzyme-linked immunosorbent assay (ELISA) (VEGF-A, VEGFR-1 - R\&D Systems Inc., Minneapolis, MN, USA; VEGF-B - Wuhan EIAab Science Co., Ltd., Wuhan, China) and chemiluminescent microparticle immunoassay (CMIA) (CA 15-3 - Abbott, Chicago, IL, USA) according to the manufacturer's protocols. In ELISA, according to the manufacturer's protocols, duplicate samples were assessed for each standard, control, and sample.

The intra-assay coefficient of variation $(\mathrm{CV} \%)$ of: $\mathrm{CA}$ $15-3$ is reported to be $2.2 \%$ at a mean concentration of $27.0 \mathrm{U} / \mathrm{mL}$, S.D.=0.6; VEGF-A to be $4.5 \%$ at a mean concentration of $235 \mathrm{pg} / \mathrm{mL}$, S.D.=10.6; VEGF-B to be $5.3 \%$; VEGFR-1 to be $2.6 \%$ at a mean concentration of $96.6 \mathrm{pg} / \mathrm{mL}$, S.D. $=2.5$.

Table 1. Characteristics of breast cancer patients and control groups: benign breast tumor and healthy women.

\begin{tabular}{|c|c|c|c|}
\hline \multicolumn{3}{|c|}{ Study group } & \multirow{2}{*}{$\frac{\text { Number of patients }}{120}$} \\
\hline \multirow{8}{*}{ 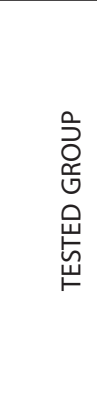 } & Breast cancer patients & carcinoma ductale & \\
\hline & Median age (range) & $58(39-83)$ & \\
\hline & \multirow{4}{*}{ Tumor stage } & 1 & 38 \\
\hline & & II & 41 \\
\hline & & III & 20 \\
\hline & & IV & 21 \\
\hline & \multirow{2}{*}{ Menopausal status: } & - premenopausal & 21 \\
\hline & & - postmenopausal & 99 \\
\hline \multirow{10}{*}{ 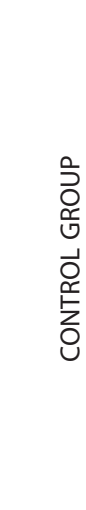 } & \multirow[t]{3}{*}{ Benign breast tumor patients } & & 28 \\
\hline & & adenoma & 10 \\
\hline & & fibroadenoma & 18 \\
\hline & Median age (range) & $48(36-71)$ & \\
\hline & \multirow[t]{2}{*}{ Menopausal status: } & - premenopausal & 10 \\
\hline & & - postmenopausal & 18 \\
\hline & Healthy women & & 32 \\
\hline & Median age (range) & $49(33-73)$ & \\
\hline & \multirow{2}{*}{ Menopausal status: } & - premenopausal & 14 \\
\hline & & - postmenopausal & 18 \\
\hline
\end{tabular}


The inter-assay coefficient of variation $(\mathrm{CV} \%)$ of: $\mathrm{CA}$ $15-3$ is reported to be $2.6 \%$ at a mean concentration of $27.0 \mathrm{U} / \mathrm{ml}$, S.D. $=0.7$; VEGF-A to be $7.0 \%$ at a mean concentration of $250 \mathrm{pg} / \mathrm{mL}$, S.D. $=17.4$; VEGF-B to be $9.5 \%$; VEGFR-1 to be $9.8 \%$ at a mean concentration of $112 \mathrm{pg} / \mathrm{mL}$, S.D. $=11.0$.

The value of intra- and inter- assay CVs were calculated by the manufacturers and enclosed in the reagent kits. The assay does not exhibit cross-reactivity or interference with numerous human cytokines and other growth factors.

Statistical analysis. Statistical analysis was performed using STATISTICA 12.0 (StatSoft, Tulsa, OK, USA). The preliminary statistical analysis (using the ShapiroWilk test) revealed that the tested parameters and tumor marker levels did not follow normal distribution. Consequently, statistical analysis between the groups was performed by using the U-Mann Whitney test, the Kruskal-Wallis test and a multivariate analysis of various data by the post-hoc Dwass-Steele-CrichlowFlinger test. The data were presented as a median and a range. Diagnostic sensitivity ( $\mathrm{Sn}$ ), specificity (Sf), and the predictive values of positive and negative test results (PPV and NPV, respectively) were calculated by using the cut-off values which were derived from the Youden's index (as a criterion for selecting the optimum cut-off point) and for each of the tested parameters were as follows: VEGF-A - $62.9 \mathrm{pg} / \mathrm{mL}$; VEGF-B - $64.1 \mathrm{pg} /$ mL; VEGFR-1 - $59.2 \mathrm{pg} / \mathrm{mL}$; CA 15-3 - $18.5 \mathrm{U} / \mathrm{mL}$.

We also defined the receiver-operating characteristics (ROC) curve for all the tested parameters and tumor markers. The construction of the ROC curves was performed using the GraphRoc program for Windows (Windows, Royal, AR, USA) and the areas under the ROC curve (AUC) were calculated to evaluate the diagnostic accuracy and to compare AUC for all tested parameters, separately and in combination with the commonly used tumor marker (CA 15-3). Statistically significant differences were defined as comparisons resulting in $p<0.05$.

\section{RESULTS}

Table 2 shows the plasma levels of the tested parameters and CA 15-3 in patients with breast cancer and in the control group. Plasma levels of all the parameters in total cancer group were statistically significantly higher (only in case of VEGF-B statistically significantly lower) when compared to the total control group (in all cases, $p<0.05$ ). In a divided control group (split into benign breast tumor patients and healthy women), statistical significance was observed in plasma levels of VEGF-A, VEGFR-1 and CA 15-3 of total cancer group compared to benign breast tumor patients, and VEGF-A, VEGF-B and CA 15-3 plasma levels in cancer patients significantly differed when compared to healthy women group.

In the patients with I, II and III stage of cancer, only VEGF-A and CA 15-3, and in patients with stage IV all the tested parameters differed significantly when compared to the total control group. When compared to the benign breast tumor group, the differences in plasma levels of VEGF-A, VEGFR-1 and CA 15-3 were statistically significant in patients with I, III and IV stage of cancer, and in patients with II stage cancer, VEGF-A and CA 15-3 plasma levels were significantly different. When compared to healthy volunteers' group, patients with I stage of cancer had only VEGF-B plasma levels significantly different, patients with II and III stage BC-

Table 2. Plasma levels of tested parameters and Cancer Antigen 15-3 in patients with breast cancer and in control group.

\begin{tabular}{|c|c|c|c|c|c|}
\hline Groups tested & & VEGF-A (pg/mL) & VEGF-B (pg/mL) & VEGFR-1 (pg/mL) & CA $15-3(\mathrm{U} / \mathrm{mL})$ \\
\hline \multirow{10}{*}{ 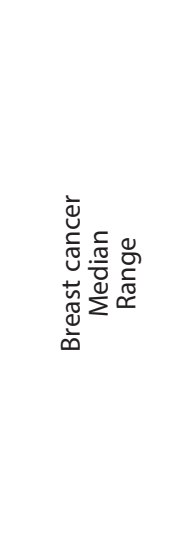 } & \multirow{2}{*}{ I stage } & $72.6 \mathrm{a} / \mathrm{c}$ & $46.1^{b}$ & $64.3^{a}$ & $16.7 \mathrm{a} / \mathrm{c} / \mathrm{d}$ \\
\hline & & $1.5-792.1$ & $19.4-115.6$ & $26.3-114.4$ & $6.2-50.3$ \\
\hline & \multirow{2}{*}{ Il stage } & $85.1 \mathrm{a} / \mathrm{b} / \mathrm{c}$ & $45.8 \mathrm{~b} / \mathrm{d}$ & 62.5 & $16.9 \mathrm{a} / \mathrm{b} / \mathrm{c} / \mathrm{d}$ \\
\hline & & $13.7-759.4$ & $34.5-78.2$ & $2.5-125.1$ & $4.4-48.1$ \\
\hline & \multirow{2}{*}{ III stage } & $82.5 \mathrm{a} / \mathrm{b} / \mathrm{c}$ & $41.6^{b}$ & $64.3^{a}$ & $26.5 \mathrm{a} / \mathrm{b} / \mathrm{c} / \mathrm{d}$ \\
\hline & & $36.5-180.3$ & $35.3-98.3$ & $36.2-138.5$ & 8.9-167.5 \\
\hline & \multirow{2}{*}{ IV stage } & $98.0 \mathrm{a} / \mathrm{b} / \mathrm{c}$ & $38.7 \mathrm{~b} / \mathrm{c} / \mathrm{d}$ & $75.7 \mathrm{a} / \mathrm{b} / \mathrm{c}$ & $45.1 \mathrm{a} / \mathrm{b} / \mathrm{c} / \mathrm{d}$ \\
\hline & & $21.6-251.7$ & $33.7-49.6$ & $47.9-99.9$ & $18.5-250.0$ \\
\hline & \multirow{2}{*}{ Total group } & $78.5 \mathrm{a} / \mathrm{b} / \mathrm{c}$ & $42.1 \mathrm{~b} / \mathrm{c}$ & $64.7 \mathrm{a} / \mathrm{c}$ & $20.0 \mathrm{a} / \mathrm{b} / \mathrm{c}$ \\
\hline & & $1.5-792.1$ & $19.4-115.6$ & $2.5-138.5$ & $4.4-250.0$ \\
\hline \multirow{6}{*}{ 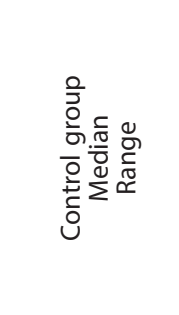 } & \multirow{2}{*}{$\begin{array}{l}\text { Benign breast } \\
\text { tumor }\end{array}$} & 19.4 & $44.8^{\mathrm{e}}$ & 46.6 & 12.8 \\
\hline & & $11.3-141.2$ & $16.7-91.5$ & $16.2-174.3$ & $4.0-20.7$ \\
\hline & \multirow{2}{*}{ Healthy women } & 46.8 & $65.5^{\mathrm{e}}$ & 55.7 & 13.4 \\
\hline & & $7.5-197.4$ & $19.4-225.2$ & $18.0-89.8$ & $6.3-28.4$ \\
\hline & \multirow{2}{*}{ Total group } & 31.4 & 53.5 & 53.7 & 13.1 \\
\hline & & $7.5-197.4$ & $16.7-225.2$ & $16.2-174.3$ & $4.0-28.4$ \\
\hline
\end{tabular}

aStatistically significant when compared to benign breast tumor patients; bStatistically significant when compared to healthy women; cStatistically significant when compared to the total control group; dStatistically significant when the breast cancer stage III or IV patients were compared to the breast cancer stage I or II patients; eStatistically significant when healthy women were compared to the patients with benign breast tumor. Abbreviations: VEGF-A - vascular endothelial growth factor A; VEGF-B - vascular endothelial growth factor B; VEGFR-1 - vascular endothelial growth factor receptor 1; CA 15-3 - cancer antigen 15-3. 
VEGF-A, VEGF-B and CA 15-3, and patients with stage IV BC - all the tested parameters plasma levels significantly different (in all cases $p<0.05$ ).

VEGF-B was the only parameter, in relation to which we have observed statistical significance in differentiation between benign breast tumor patients and healthy women group.

Table 3 shows the sensitivity (Sn), specificity (Sf), positive predictive value (PPV) and negative predictive value (NPV) of the investigated parameters and CA 15-3. We indicated that the Sn of all the tested parameters in the total cancer group was the highest for VEGF-B (90\%). Among all the parameters, the highest $\mathrm{Sn}$ in stages I and II of cancer was observed also for VEGF-B $(86.8 \% ; 92.7 \%$, respectively), in case of III stage for VEGFR-1 (95\%), and in case of IV stage for VEGF-B and VEGFR-1 (both 100\%).

The diagnostic Sf of the tested parameters was the highest for VEGF-A (76.7\%), but lower than for the commonly used tumor marker (95\%). The predictive value of a positive test result (PPV) in the total group of BC patients was the highest for VEGF-A (84.8\%), but it was still lower than PPV of CA 15-3 (95.9\%). Among all the tested parameters, the highest PPV values in all stages of cancer were observed also for VEGF-A $(60 \%$; $67.4 \% ; 50 \% ; 50 \%$, respectively), but also they were lower than PPV of CA 15-3.

The highest predictive value of a negative test result (NPV) in the total group of BC was shown by VEGF-B $(66.7 \%)$. The highest NPV in stages I, II and IV of BC was observed also for VEGF-B $(82.8 \% ; 88.9 \% ; 100 \%$, respectively), and in stage III of cancer for VEGFR-1 $(97.4 \%)$.

Combined analysis of tested parameters and CA 15-3 resulted in increase of Sn and NPV in almost all cases. The most favorable combination proved to be CA 15-3 + VEGF-A in total BC group.

Table 3. Diagnostic criteria of tested parameters and CA 15-3 in patients with breast cancer.

\begin{tabular}{|c|c|c|c|c|c|c|}
\hline \multirow{2}{*}{ Tested parameters } & \multirow{2}{*}{ Diagnostic criteria (\%) } & \multicolumn{5}{|l|}{ Breast cancer } \\
\hline & & Total group & I stage & II stage & III stage & IV stage \\
\hline \multirow{4}{*}{ CA 15-3 } & Sn & 58.3 & 39.5 & 46.3 & 75.0 & 90.5 \\
\hline & Sf & 95.0 & 95.0 & 95.0 & 95.0 & 95.0 \\
\hline & PPV & 95.9 & 83.3 & 86.4 & 83.3 & 86.4 \\
\hline & NPV & 53.3 & 71.3 & 72.2 & 91.9 & 96.6 \\
\hline \multirow{4}{*}{ VEGF-A } & Sn & 65.0 & 55.3 & 70.7 & 70.0 & 66.7 \\
\hline & Sf & 76.7 & 76.7 & 76.7 & 76.7 & 76.7 \\
\hline & PPV & 84.8 & 60.0 & 67.4 & 50.0 & 50.0 \\
\hline & NPV & 52.3 & 73.0 & 79.3 & 88.5 & 86.8 \\
\hline \multirow{4}{*}{ VEGF-B } & Sn & 90.0 & 86.8 & 92.7 & 80.0 & 100.0 \\
\hline & Sf & 40.0 & 40.0 & 40.0 & 40.0 & 40.0 \\
\hline & PPV & 75.0 & 47.8 & 51.4 & 30.8 & 36.8 \\
\hline & NPV & 66.7 & 82.8 & 88.9 & 85.7 & 100.0 \\
\hline \multirow{4}{*}{ VEGFR-1 } & Sn & 60.0 & 65.8 & 51.2 & 95.0 & 100.0 \\
\hline & Sf & 63.3 & 63.3 & 63.3 & 63.3 & 63.3 \\
\hline & PPV & 76.6 & 53.2 & 48.8 & 45.2 & 40.5 \\
\hline & NPV & 44.2 & 74.5 & 65.5 & 97.4 & 86.4 \\
\hline \multirow{4}{*}{ CA $15-3$ + VEGF-A } & Sn & 83.3 & 71.1 & 78.1 & 100.0 & 100.0 \\
\hline & Sf & 73.3 & 73.3 & 73.3 & 73.3 & 73.3 \\
\hline & PPV & 86.2 & 62.8 & 66.7 & 55.6 & 56.7 \\
\hline & NPV & 68.8 & 80.0 & 83.0 & 100.0 & 100.0 \\
\hline \multirow{4}{*}{ CA $15-3$ + VEGF-B } & Sn & 91.7 & 86.8 & 95.1 & 85.0 & 100.0 \\
\hline & Sf & 38.3 & 38.3 & 38.3 & 38.3 & 38.3 \\
\hline & PPV & 74.8 & 47.1 & 51.3 & 31.5 & 36.2 \\
\hline & NPV & 69.7 & 82.1 & 92.0 & 88.5 & 100.0 \\
\hline \multirow{4}{*}{ CA $15-3$ + VEGFR-1 } & Sn & 83.3 & 79.0 & 73.2 & 95.0 & 100.0 \\
\hline & $\mathrm{Sf}$ & 61.7 & 61.7 & 61.7 & 61.7 & 61.7 \\
\hline & PPV & 81.3 & 56.6 & 56.6 & 45.2 & 47.7 \\
\hline & NPV & 64.9 & 82.2 & 77.1 & 97.4 & 100.0 \\
\hline
\end{tabular}

Abbreviations: Sn, sensitivity; Sf, specificity; PPV, positive predictive value; NPV, negative predictive value; VEGF-A, vascular endothelial growth factor A; VEGF-B, vascular endothelial growth factor B; VEGFR-1, vascular endothelial growth factor receptor 1; CA 15-3, cancer antigen 15-3. 


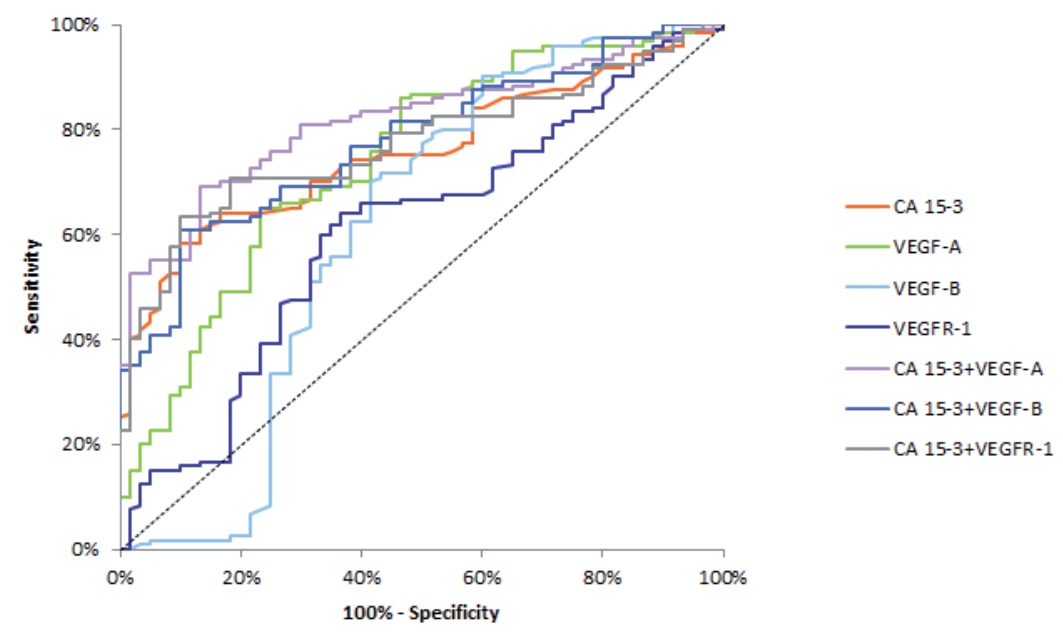

Figure 1. Diagnostic criteria of receiver operating characteristics curve for the tested parameters alone and in combination with cancer antigen 15-3 in the total breast cancer group.

The relationship between the diagnostic $\mathrm{Sn}$ and Sf is illustrated by the ROC curve. The area under the ROC curve (AUC) indicates the clinical usefulness of a tumor marker and its diagnostic power. All data relating to the AUC in the total BC group are included in Table 4. Graphic version of the ROC curve for all parameters tested individually and in combination with commonly used tumor marker in the entire BC group is shown in Fig. 1. We noticed that the VEGF-A area under the ROC curve (0.742) in the total group of breast cancer was the largest between all the individually tested parameters, only slightly smaller than CA 15-3 (0.757). In case of all the stages of BC (I-IV), AUC was the largest also for VEGF-A (0.678; 0.768; 0.763; 0.788, respectively), and only in $\mathrm{BC}$ stages III and IV, AUC of CA $15-3$ was larger (0.869; 0.967, respectively).

Combining of the tested parameters and CA 15-3 resulted in an increase of AUC in all cases. The most favorable combinations, not only in stages I-IV, but also in total cancer group, proved to be VEGF-A and CA 15-3. The AUCs for the tested parameters, similar to commonly used tumor markers, were statistically significantly larger in comparison to $\mathrm{AUC}=0.5$, which is a borderline of the diagnostic usefulness of the test $(p<0.05$ in all cases).

\section{DISCUSSION}

Angiogenesis and lymphangiogenesis are crucial for tumor progression and nutrition. Vascular endothelial growth factor family members and their receptors have a direct effect on endothelial cell proliferation and migration and are potent stimulators of these processes. Early diagnosis and determination of cancer stage allows to increase the survival of patients with breast cancer by indicating effective treatment methods. According to many reports regarding the usefulness of tumor markers not only in breast cancer, it is very important that the diagnosis is not limited to diagnostic imaging (Lawicki et al., 2013; Ławicki et al., 2016; Zajkowska et al., 2016; Będkowska et al., 2017).

In the present study we investigated the usefulness of assessing plasma levels of VEGF-A, VEGF-B and VEGFR-1, individually and in combination with CA 15-3 (a commonly used tumor marker) in breast cancer patients, not only in the total group of patients, but also in particular cancer stage groups (stages I, II, III and IV).

Statistically significant plasma overexpression and high gene expression of VEGF-A, VEGF-B and VEGFR-1 have been detected in patients suffering from many types of tumors, also breast cancer (Takahashi, 2011; De Falco, 2012; Thielemann et al., 2013; Lawicki et al., 2013; Zyła et al., 2014; Bry et al., 2014; Lawicki et al., 2016). We have demonstrated statistically significantly higher plasma concentrations of tested parameters in $\mathrm{BC}$ patients compared to control groups. Comparable results for VEGF-A and VEGFR-1 were obtained by Thielemann and others (Thielemann et al., 2013) in breast cancer patients, but this study the control consisted only of the healthy subjects, and the cancer group consisted of patients with stages I-III BC (TNM classification). Both, VEGF-A and VEGFR-1 plasma levels were statistically altered in all stages of cancer when compared to healthy subjects. In our research, only VEGF-A showed statistical significance when compared to healthy subjects, in stages II-IV. This discrepancy might be related to different concentrations of the tested parameters obtained in healthy controls group. Better results were obtained in comparison to benign breast tumor patients, where both parameters showed statistical significance in all stages of cancer (with exception of stage II in case of VEGFR-1). In opposition to our findings, the results obtained by Kotowicz and others (Kotowicz et al., 2017), showed that concentrations of VEGF-A and VEGFR-1 in plasma did not vary significantly between cancer patients and healthy controls, but their work concerned different type of tumor (endometrial cancer). Our work is first to our knowledge, which addresses the plasma concentrations of VEGF-B cytokine in cancer as a potential marker. We have found only publications which revealed higher gene expression of VEGF-B in cancer and one publication with concentrations of this cytokine in aqueous humour of patients with glaucoma (Yang et al., 2015; Chen et al., 2015). According to the results obtained by Chen and others (Chen et al., 2015), the concentrations of VEGF-B in the serum and aqueous humour of glaucoma glaucoma were much higher than we observed in our study (114 and $104 \mathrm{pg} /$ $\mathrm{mL})$. VEGF-B was also the only parameter in our study, for which we have observed statistical significance in differentiation between benign breast tumor patients and healthy women group.

Sensitivity ( $\mathrm{Sn}$ ) measures the proportion of correctly identified positives. In this study, VEGF-B displayed the highest $\mathrm{Sn}$ in the total group of breast cancer patients. To our knowledge, this study is the first one estimating not only concentrations, but also diagnostic utility of VEGF-B. VEGF-A and VEGFR-1 also had high Sn as BC markers (higher than commonly used tumor marker) not only in total cancer group, but also in individual stages I to IV. The results obtained by Kotowicz and others (Kotowicz et al., 2017) for I stage of endometrial cancer showed VEGF-A Sn similar to ours (56\%), but much more lower Sn for VEGFR-1 (16\%). This 
Table 4. Diagnostic criteria of receiver operating characteristics (ROC) curve for tested parameters in all stages of breast cancer.

\begin{tabular}{|c|c|c|c|c|}
\hline \multirow{2}{*}{ Tested parameters } & \multicolumn{4}{|c|}{ ROC criteria in breast cancer (I stage) } \\
\hline & AUC & SE & 95\% C.I. (AUC) & $p(\mathrm{AUC}=0.5)$ \\
\hline CA $15-3$ & 0.648 & 0.061 & $(0.528-0.768)$ & 0.02 \\
\hline VEGF-A & 0.678 & 0.057 & $(0.566-0.790)$ & $<0.01$ \\
\hline VEGF-B & 0.576 & 0.058 & $(0.463-0.690)$ & 0.19 \\
\hline VEGFR-1 & 0.610 & 0.059 & $(0.493-0.726)$ & 0.07 \\
\hline CA $15-3+$ VEGF-A & 0.723 & 0.058 & $(0.610-0.836)$ & $<0.001$ \\
\hline CA $15-3+$ VEGF-B & 0.680 & 0.058 & $(0.567-0.793)$ & $<0.01$ \\
\hline CA $15-3$ + VEGFR-1 & 0.665 & 0.060 & $(0.547-0.783)$ & 0.01 \\
\hline \multirow{2}{*}{ Tested parameters } & \multicolumn{4}{|c|}{ ROC criteria in breast cancer (II stage) } \\
\hline & AUC & SE & 95\% C.I. (AUC) & $p(\mathrm{AUC}=0.5)$ \\
\hline CA $15-3$ & 0.697 & 0.057 & $(0.586-0.808)$ & $<0.001$ \\
\hline VEGF-A & 0.768 & 0.047 & $(0.675-0.860)$ & $<0.001$ \\
\hline VEGF-B & 0.609 & 0.057 & $(0.497-0.720)$ & 0.06 \\
\hline VEGFR-1 & 0.527 & 0.061 & $(0.407-0.647)$ & 0.66 \\
\hline CA $15-3$ + VEGF-A & 0.766 & 0.051 & $(0.667-0.866)$ & $<0.001$ \\
\hline CA $15-3$ + VEGF-B & 0.739 & 0.050 & $(0.640-0.837)$ & $<0.001$ \\
\hline CA $15-3$ + VEGFR-1 & 0.707 & 0.057 & $(0.595-0.819)$ & $<0.001$ \\
\hline \multirow{2}{*}{ Tested parameters } & \multicolumn{4}{|c|}{ ROC criteria in breast cancer (III stage) } \\
\hline & AUC & SE & 95\% C.I. (AUC) & $p(\mathrm{AUC}=0.5)$ \\
\hline CA $15-3$ & 0.869 & 0.056 & $(0.760-0.978)$ & $<0.001$ \\
\hline VEGF-A & 0.763 & 0.053 & $(0.658-0.867)$ & $<0.001$ \\
\hline VEGF-B & 0.578 & 0.067 & $(0.447-0.709)$ & 0.24 \\
\hline VEGFR-1 & 0.623 & 0.074 & $(0.477-0.768)$ & 0.10 \\
\hline CA $15-3+$ VEGF-A & 0.924 & 0.029 & $(0.867-0.981)$ & $<0.001$ \\
\hline CA $15-3+$ VEGF-B & 0.849 & 0.062 & $(0.728-0.970)$ & $<0.001$ \\
\hline CA $15-3$ + VEGFR-1 & 0.905 & 0.046 & $(0.815-0.995)$ & $<0.001$ \\
\hline \multirow{2}{*}{ Tested parameters } & \multicolumn{4}{|c|}{ ROC criteria in breast cancer (IV stage) } \\
\hline & AUC & SE & $95 \%$ C.I. (AUC) & $p(\mathrm{AUC}=0.5)$ \\
\hline CA $15-3$ & 0.967 & 0.017 & $(0.934-0.999)$ & $<0.001$ \\
\hline VEGF-A & 0.788 & 0.057 & $(0.676-0.901)$ & $<0.001$ \\
\hline VEGF-B & 0.716 & 0.056 & $(0.606-0.825)$ & $<0.001$ \\
\hline VEGFR-1 & 0.744 & 0.054 & $(0.638-0.849)$ & $<0.001$ \\
\hline CA $15-3+$ VEGF-A & 0.986 & 0.010 & $(0.966-1.005)$ & $<0.001$ \\
\hline CA $15-3+$ VEGF-B & 0.962 & 0.018 & $(0.926-0.998)$ & $<0.001$ \\
\hline CA $15-3$ + VEGFR-1 & 0.971 & 0.015 & $(0.942-1.000)$ & $<0.001$ \\
\hline \multirow{2}{*}{ Tested parameters } & \multicolumn{4}{|c|}{ ROC criteria in total breast cancer group } \\
\hline & AUC & SE & 95\% C.I. (AUC) & $p(\mathrm{AUC}=0.5)$ \\
\hline CA 15-3 & 0.757 & 0.035 & $(0.688-0.826)$ & $<0.001$ \\
\hline VEGF-A & 0.742 & 0.040 & $(0.665-0.819)$ & $<0.001$ \\
\hline VEGF-B & 0.612 & 0.052 & $(0.510-0.714)$ & 0.03 \\
\hline VEGFR-1 & 0.607 & 0.045 & $(0.519-0.695)$ & 0.02 \\
\hline CA $15-3$ + VEGF-A & 0.817 & 0.031 & $(0.757-0.878)$ & $<0.001$ \\
\hline CA $15-3+$ VEGF-B & 0.778 & 0.034 & $(0.711-0.845)$ & $<0.001$ \\
\hline CA $15-3$ + VEGFR-1 & 0.773 & 0.034 & $(0.706-0.840)$ & $<0.001$ \\
\hline
\end{tabular}

$p$, AUC statistically significantly larger compared to $A \cup C=0.5$. Abbreviations: $A \cup C$, area under curve; ROC, receiver operating characteristics; $S . E$., standard error; 95\% C.I., 95\% confidence interval; VEGF-A, vascular endothelial growth factor A; VEGF-B, vascular endothelial growth factor B; VEGFR-1, vascular endothelial growth factor receptor 1; CA 15-3, cancer antigen 15-3. 
discrepancy might be explained by a different type of the tumor examined. In a study by Wu ansd others (Wu et al., 2017) concerning pleural effusions, where VEGF-A was assessed as a prognostic factor, the results were very promising (VEGF-A Sn of $76 \%$ ), which shows that VEGF-A might be a useful marker not only in cancer differentiation.

Specificity ( $\mathrm{Sf}$ ) measures the proportion of correctly identified negatives. In this study, in the total group of breast cancer patients, VEGF-A displayed the highest Sf among the tested parameters, but it was still lower than CA 15-3 Sf. We have obtained a higher Sf for VEGF-A and similar Sf for CA 15-3 in our previous work concerning breast cancer (Lawicki et al., 2016), but in that study, we used a different method for calculating Sf (95th percentile, not Youden index as currently), which may account for the differences in the ratio of diagnostic sensitivity and specificity. In the study of Wu and others (Wu et al., 2017), Sf for VEGF-A was also high (84.2\%).

Our results show that in all the groups of BC patients, VEGF-A has the highest PPV values among all the tested parameters, but they are still lower than the commonly used tumor marker. In a previously published studies, PPV for VEGF-A were also very high (Lawicki et al., 2016; Zajkowska et al., 2016). In the total $\mathrm{BC}$ group and in different stages of $\mathrm{BC}$, the predictive value of a negative test result (NPV) was usually the highest for VEGF-B. Since this study is the first, to our knowledge, to contain not only concentrations, but also a wide statistical analysis of VEGF-B plasma levels, we are not able to compare our results to the work of other authors.

The most important criterion for tumor markers is the Sn/Sf diagram - ROC curve. The diagnostic power (AUC) represents the overall accuracy of a test, with the value approaching 1.0 indicating a perfect $\mathrm{Sn}$ and Sf. Our results showed that VEGF-A $(0.742)$ had the highest AUC value of all the tested parameters in the total group of BC patients, but slightly lower than CA 15-3. Similar results were obtained in a study by Kotowicz and others (Kotowicz et al., 2017), where AUC value was extremely high and approached value of 0.904. AUC for VEGFR-1 (0.743) was also larger than ours (0.607), but our analysis was performed with use of a combined control group (not only healthy subjects, but also benign breast tumor patients). Similar results were obtained by $\mathrm{Wu}$ and others (Wu et al., 2017), where VEGF-A had AUC $=0.728$.

Our research group is the only one evaluating the diagnostic usefulness of parameters in such an advanced way (combined analysis of all the tested parameters with the commonly used tumor marker). We have found only one study, concerning endometrial cancer, conducted by Kotowicz and others (Kotowicz et al., 2017), where, in I stage of cancer, $\mathrm{Sn}$ was assessed for a combined CA 125 and VEGF-A, and obtained $68 \%$, which is comparable to the result of our combined analysis of CA 15-3 with VEGF-A ( $\mathrm{Sn}=71.1 \%$ in I stage of $\mathrm{BC})$.

What is important, in future diagnosis, a combined analysis of the tested parameters and CA 15-3 can be the best way to improve the detection of breast cancer, because most of the currently studiedparameters are nonspecific and should only be used in panel to improve their sensitivity.

\section{CONCLUSIONS}

Early detection of breast cancer in patients is of utter importance. Our present results indicate the usefulness and high diagnostic power of all the tested parameters in the detection of breast cancer. Among the tested parameters, VEGF-A appeared to be the best candidate for cancer diagnostics (superior to the commonly used tumor marker - CA 15-3) especially in the stages I and II of BC. The combined analysis of the tested parameters and CA 15-3 resulted in an increase in $\mathrm{Sn}$ and AUC values, which provides hope for developing a new panel of biomarkers that may be used in the diagnosis of $\mathrm{BC}$ in the future.

\section{Conflicts of interest}

None declared.

\section{Ethics approval and informed consent}

The study was approved by the local Ethics Committee in Medical University of Białystok (R-I002/70/2015). All the patients gave their informed consent for participation in the study.

\section{Ethical declaration}

This work was conducted in accordance with the Declaration of Helsinki (1964).

\section{Contribution Statement}

MZ conceived the idea for the study. MZ, SL, MSZ contributed to the design of the research. All authors were involved in data collection and analyzed the data. MZ coordinated funding for the project. All authors edited and approved the final version of the manuscript.

\section{Acknowledgements of Finanacial Support}

This research was financed by Grants for Medical University of Białystok (N/ST/MN/17/002/2207, N/ $\mathrm{ST} / \mathrm{MN} / 18 / 001 / 2207)$ from the Polish Ministry of Science and Higher Education.

\section{REFERENCES}

Bry M, Kivelä R, Leppänen VM, Alitalo K (2014) Vascular endothelial growth factor-B in physiology and disease. Physiol Rev 94: 779-794. doi: 10.1152 / physrev.00028.2013

Będkowska GE, Gacuta E, Zajkowska M, Glażewska EK, Osada J, Szmitkowski M, Chrostek L, Dabrowska M, Lawicki S (2017) Plasma levels of MMP-7 and TIMP-1 in laboratory diagnostics and differentiation of selected histological types of epithelial ovarian cancers. J Ovarian Res 10: 39. doi: 10.1186/s13048-017-0338-z

Caballero B, Sherman SJ, Falk T (2017) Insights into the mechanisms involved in protective effects of VEGF-B in dopaminergic neurons. Parkinsons Dis 2017: 4263795. doi: 10.1155/2017/4263795

Carmeliet P, Ruiz de Almodovar C, Carmen ReA (2013) VEGF ligands and receptors: implications in neurodevelopment and neurodegeneration. Cell Mol Life Sci 70: 1763-1778. doi: 10.1007/s00018-0131283-7

Chen S, Zhou M, Wang W, Wu H, Yu X, Huang W, Gao X, Wang J, Li X, Du S, Ding X, Zhang X (2015) Levels of angiogenesis-related vascular endothelial growth factor family in neovascular glaucoma eyes. Acta Ophthalmol 93: e556-e560. doi: 10.1111/aos.12624

De Falco S (2012) The discovery of placenta growth factor and its biological activity. Exp Mol Med 44: 1-9. doi: 10.3858/ emm.2012.44.1.025

Egeblad M, Werb Z (2002) New functions for the matrix metalloproteinases in cancer progression. Nat Rev Cancer 2: 161-174. doi: $10.1038 / \operatorname{nrc} 745$

Harris L, Fritsche H, Mennel R, Norton L, Ravdin P, Taube S, Somerfield MR, Hayes DF, Bast RC, American Society of Clinical Oncology (2007) American Society of Clinical Oncology 2007 update of recommendations for the use of tumor markers in breast cancer. $J$ Clin Oncol 25: 5287-5312. doi: 10.1200/JCO.2007.14.2364

Kotowicz B, Fuksiewicz M, Jonska-Gmyrek J, Berezowska A, Radziszewski J, Bidzinski M, Kowalska M (2017) Clinical significance of pretreatment serum levels of VEGF and its receptors, IL- 8, and their 
prognostic value in type I and II endometrial cancer patients. PLoS One 12: e0184576. doi: 10.1371/journal.pone.0184576

Leung DW, Cachianes G, Kuang WJ, Goeddel DV, Ferrara N (1989) Vascular endothelial growth factor is a secreted angiogenic mitogen. Science 246: 1306-1309

Ławicki S, Będkowska GE, Gacuta-Szumarska E, Szmitkowski M (2013) The plasma concentration of VEGF, HE4 and CA125 as a new biomarkers panel in different stages and sub-types of epithelial ovarian tumors. J Ovarian Res 6: 45. doi: 10.1186/1757-2215-6-45

Ławicki S, Mroczko B, Szmitkowski M (2004) Tumor markers of breast cancer. Postepy Hig Med Dosw (Online) 58: 292-300

Ławicki S, Zajkowska M, Głażewska EK, Będkowska GE, Szmitkowski M (2016) Plasma levels and diagnostic utility of VEGF, MMP-9, and TIMP-1 in the diagnosis of patients with breast cancer. Onco Targets Ther 9: 911-919. doi: 10.2147/OT'T.S99959

Oeffinger KC, Fontham ET, Etzioni R, Herzig A, Michaelson JS, Shih YC, Walter LC, Church TR, Flowers CR, LaMonte SJ, Wolf AM, DeSantis C, Lortet-Tieulent J, Andrews K, Manassaram-Baptiste D, Saslow D, Smith RA, Brawley OW, Wender R, American Cancer Society (2015) Breast cancer screening for women at average risk: 2015 guideline update from the American Cancer Society. JAMA 314: 1599-1614. doi: 10.1001/jama.2015.12783

Siegel RL, Miller KD, Jemal A (2018) Cancer statistics, 2018. CA Cancer J Clin 68: 7-30. doi: 10.3322/caac. 21442

Takahashi S (2011) Vascular endothelial growth factor (VEGF), VEGF receptors and their inhibitors for antiangiogenic tumor therapy. Biol Pharm Bull 34: 1785-1788

Thielemann A, Baszczuk A, Kopczyński Z, Kopczyński P, Grodecka-Gazdecka S (2013) Clinical usefulness of assessing VEGF and soluble receptors sVEGFR-1 and sVEGFR-2 in women with breast cancer. Ann Agric Environ Med 20: 293-297

Torre LA, Bray F, Siegel RL, Ferlay J, Lortet-Tieulent J, Jemal A (2015) Global cancer statistics, 2012. CA Cancer J Clin 65: 87-108. doi: $10.3322 /$ caac. 21262

Weryńska B, Dziegiel P, Jankowska R (2009) Role of lymphangiogenesis in lung cancer. Folia Histochem Cytobiol 47: 333-342. doi: 10.2478/ v10042-009-0090-3

Wu DW, Chang WA, Liu KT, Yen MC, Kuo PL (2017) Vascular endothelial growth factor and protein level in pleural effusion for differentiating malignant from benign pleural effusion. Oncol Lett 14: 3657-3662. doi: 10.3892/ol.2017.6631

Yang X, Zhang Y, Hosaka K, Andersson P, Wang J, Tholander F, Cao Z, Morikawa H, Tegnér J, Yang Y, Iwamoto H, Lim S, Cao Y (2015) VEGF-B promotes cancer metastasis through a VEGF-A-independent mechanism and serves as a marker of poor prognosis for cancer patients. Proc Natl Acad Sci U S A 112: E2900-E2909. doi: $10.1073 /$ pnas. 1503500112

Zajkowska M, Głażewska EK, Będkowska GE, Chorąży P, Szmitkowski M, Lawicki S (2016) Diagnostic power of vascular endothelial growth factor and macrophage colony-stimulating factor in breast cancer patients based on ROC analysis. Mediators Inflamm 2016: 5962946. doi: 10.1155/2016/5962946

Żyła MM, Kostrzewa M, Litwińska E, Szpakowski A, Wilczyński JR, Stetkiewicz T (2014) The role of angiogenic factors in endometrial cancer. Pry Menopauzalny 13: 122-126. doi: 10.5114/pm.2014.42714 